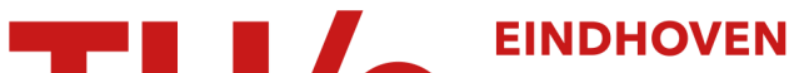 UNIVERSITY OF TECHNOLOGY
}

\section{Calculation of thermodynamic properties of finite Bose- Einstein systems}

Citation for published version (APA):

Borrmann, P., Harting, J. D. R., Mülken, O., \& Hilf, E. (1999). Calculation of thermodynamic properties of finite Bose-Einstein systems. Physical Review A : Atomic, Molecular and Optical Physics, 60(2), 1519-1522. https://doi.org/10.1103/PhysRevA.60.1519

DOI:

10.1103/PhysRevA.60.1519

Document status and date:

Published: 01/01/1999

Document Version:

Publisher's PDF, also known as Version of Record (includes final page, issue and volume numbers)

Please check the document version of this publication:

- A submitted manuscript is the version of the article upon submission and before peer-review. There can be important differences between the submitted version and the official published version of record. People interested in the research are advised to contact the author for the final version of the publication, or visit the $\mathrm{DOI}$ to the publisher's website.

- The final author version and the galley proof are versions of the publication after peer review.

- The final published version features the final layout of the paper including the volume, issue and page numbers.

Link to publication

\section{General rights}

Copyright and moral rights for the publications made accessible in the public portal are retained by the authors and/or other copyright owners and it is a condition of accessing publications that users recognise and abide by the legal requirements associated with these rights.

- Users may download and print one copy of any publication from the public portal for the purpose of private study or research.

- You may not further distribute the material or use it for any profit-making activity or commercial gain

- You may freely distribute the URL identifying the publication in the public portal.

If the publication is distributed under the terms of Article $25 \mathrm{fa}$ of the Dutch Copyright Act, indicated by the "Taverne" license above, please follow below link for the End User Agreement:

www.tue.nl/taverne

Take down policy

If you believe that this document breaches copyright please contact us at:

openaccess@tue.nl

providing details and we will investigate your claim. 


\title{
Calculation of thermodynamic properties of finite Bose-Einstein systems
}

\author{
Peter Borrmann,* Jens Harting, Oliver Mülken, and Eberhard R. Hilf \\ Department of Physics, Carl von Ossietzky University, Oldenburg, D-26111 Oldenburg, Germany
}

(Received 26 August 1998)

\begin{abstract}
We derive an exact recursion formula for the calculation of thermodynamic functions of finite systems obeying Bose-Einstein statistics. The formula is applicable for canonical systems where the particles can be treated as noninteracting in some approximation, e.g., like Bose-Einstein condensates in magnetic traps. The numerical effort of our computation scheme grows only linearly with the number of particles. As an example, we calculate the relative ground-state fluctuations and specific heats for ideal Bose gases with a finite number of particles enclosed in containers of different shapes. [S1050-2947(99)04203-1]
\end{abstract}

PACS number(s): 03.75.Fi, 05.30.Jp, 32.80.Pj

With the observation of Bose-Einstein condensation (BEC) of magnetically [1-3] and optically [4] trapped atoms, new insights into the nature of this state of matter have been given. The experimental situation is in all cases quite different from the ideal gas treated within the grandcanonical ensemble, which is the standard textbook example. First, the number of particles within the traps is fixed and finite, which suggests a canonical or microcanonical treatment of the systems. Second, the confining trap potentials greatly influence the condensate properties. Third, although the trapped gases are quite dilute, the validity of the treatment as noninteracting particle gases has to be checked from case to case.

Even within the approximation of noninteracting particles, the calculation of the thermodynamic properties of the Bose-Einstein systems remains a difficult mathematical problem. Recently, some approximate methods to calculate the fluctuation of the ground-state occupation number in a trapped Bose-Einstein condensate have been developed [5-8]. Here we present an exact method to calculate all thermodynamic quantities of finite canonical Bose systems, given the one-particle density of states.

As the starting point, we utilize the recursive formula of the canonical partition function for a system of $N$ noninteracting bosons as given in [9]

$$
Z_{N}(\beta)=\frac{1}{N} \sum_{k=1}^{N} Q_{k}(\beta) Z_{N-k}(\beta),
$$

where $Q_{k}(\beta)=Z_{1}(k \beta)=\Sigma_{i} \exp \left(-k \beta \epsilon_{i}\right)$ is the one-particle partition function at the temperatures $k \beta$ and $Z_{0}(\beta)=1$. The microcanonical partition $\Gamma_{N}(E)$ can be calculated by an inverse Laplace-transform of Eq. (1) and is given by

$$
\begin{aligned}
\Gamma_{N}(E) & =\frac{1}{N} \sum_{k=1}^{N} \frac{1}{2 \pi i} \int_{c-i \infty}^{c+i \infty} d \beta \exp (\beta E) Q_{k}(\beta) Z_{N-k}(\beta) \\
& =\frac{1}{N} \sum_{k=1}^{N} \int_{0}^{E} d E^{\prime} \Gamma_{1}^{k}\left(E^{\prime}\right) \Gamma_{N-k}\left(E-E^{\prime}\right),
\end{aligned}
$$

\footnotetext{
*Author to whom correspondence should be addressed. Electronic address: borrmann@uni-oldenburg.de
}

where $\Gamma_{1}^{k}(E)$ is the inverse Laplace transform of $Q_{k}(\beta)$ and $\Gamma_{0}(E)=\delta(E)$. A similar, slightly less general, equation has recently been derived by Weiss and Wilkens [10].

Equation (1) can be used to calculate all thermodynamic quantities by appropriate differentiation of $\ln Z_{N}$. However, in any case $Z_{N}$ occurs as a normalization factor and has to be calculated explicitly. This turns out to be a major drawback. First, the numerical effort to calculate $Z_{N}$ grows with the square of the particle number $N$. Moreover, since $Z_{N}(\beta)$ grows exponentially with $N$, multiple precision arithmetic is required for proper calculation. We will present a method avoiding these difficulties.

To ease our derivations we rewrite $Z_{N}(\beta)$ utilizing the $\mathcal{Z}$ transform and define

$$
\begin{aligned}
& \mathcal{Z}(Z)=F(x)=\sum_{k=0}^{\infty} \frac{Z_{k}(\beta)}{x^{k}}, \\
& \mathcal{Z}(Q)=G(x)=\sum_{k=0}^{\infty} \frac{Q_{k}(\beta)}{x^{k}},
\end{aligned}
$$

where we define $Q_{0}(\beta)=0$. Taking advantage of the basic properties of the $\mathcal{Z}$ transform, Eq. (1) can be written in the form

$$
-x \frac{d}{d x} F(x)=F(x) G(x),
$$

yielding $^{1}$

$$
F(x)=\exp \left(\sum_{k=1}^{\infty} \frac{Q_{k}(\beta)}{k} x^{-k}\right)
$$

Applying the inverse $\mathcal{Z}$ transform, we may write $Z_{N}(\beta)$ as

$$
Z_{N}(\beta)=\frac{1}{2 \pi i} \int_{C} F(x) x^{N-1} d x
$$

\footnotetext{
${ }^{1}$ Note that $F(x)$ is closely related to the grand-canonical partition function.
} 
where $C:=\{z \in C:|x|=r\}$ and $r$ has to satisfy the condition $\left|Z_{N}(\beta)\right| \leqslant \exp (r N)$. Alternatively we may write

$$
Z_{N}(\beta)=\left.\frac{1}{N !} \frac{d^{N}}{d x^{N}} F(1 / x)\right|_{x=0}
$$

Using Eq. (8), the number of particles with energy $\epsilon_{i}$ can be calculated by

$$
\begin{aligned}
\eta_{i}(N, \beta) & =\frac{-1}{\beta} \frac{\partial}{\partial \epsilon_{i}} \ln Z_{N}(\beta) \\
& =\frac{1}{Z_{N}(\beta)} \sum_{k=1}^{N} \exp \left(-\beta k \epsilon_{i}\right) Z_{N-k}(\beta) .
\end{aligned}
$$

Some reordering yields

$$
\eta_{i}(N+1, \beta)=\frac{Z_{N}(\beta)}{Z_{N+1}(\beta)} \exp \left(-\beta \epsilon_{i}\right)\left[\eta_{i}(N, \beta)+1\right] .
$$

Since the particle number is a conserved quantity in the canonical ensemble, the direct calculation of the normalization factor can be omitted by using the relation

$$
\frac{Z_{N}(\beta)}{Z_{N+1}(\beta)}=\frac{N+1}{\sum_{i=0}^{\infty} \exp \left(-\beta \epsilon_{i}\right)\left[\eta_{i}(N, \beta)+1\right]} .
$$

For Fermi systems, the recursion formula

$$
\eta_{i}(N+1, \beta)=\frac{Z_{N}(\beta)}{Z_{N+1}(\beta)} \exp \left(-\beta \epsilon_{i}\right)\left[1-\eta_{i}(N, \beta)\right]
$$

with
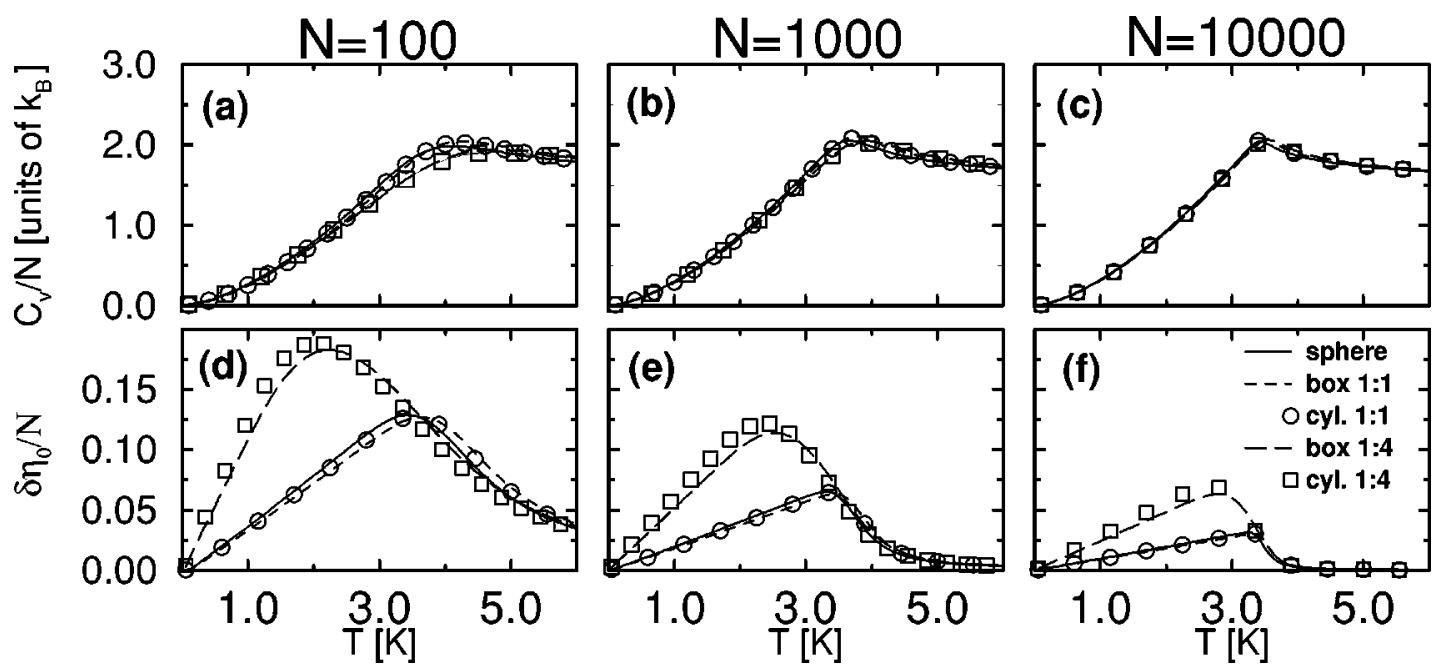

can be derived in a similar manner. In practice, only a limited number of energy levels has to be taken into account, since the occupation probability rapidly decreases with increasing energy eigenvalues. Equations (10) and (11) are extremely useful in practical calculations. The numerical effort to calculate the occupation numbers grows only linear with the number of particles. Moreover, only a moderate arithmetic precision is required. Having the occupation probabilities at hand, the energy expectation value is given by

$$
E(N, \beta)=\sum_{i=0}^{\infty} \epsilon_{i} \eta_{i}(N, \beta)
$$

The calculation of the fluctuation of the occupation probabilities $\delta \eta_{i}(N, \beta)$ is a little bit more complicated and contains another recursion:

$$
\begin{aligned}
{\left[\delta \eta_{i}(N+1, \beta)\right]^{2}=} & \frac{1}{\beta^{2}} \frac{\partial^{2}}{\partial \epsilon_{i}^{2}} \ln \left[Z_{N+1}(\beta)\right] \\
= & \frac{-1}{\beta} \frac{\partial}{\partial \epsilon_{i}} \eta_{i}(N+1, \beta) \\
= & \frac{Z_{N}(\beta)}{Z_{N+1}(\beta)} \exp \left(-\beta \epsilon_{i}\right)\left\{\delta^{2} \eta_{i}(N, \beta)\right. \\
& +\left[\eta_{i}(N+1, \beta)+1\right] \\
& \left.\times\left[\eta_{i}(N, \beta)-\eta_{i}(N+1, \beta)+1\right]\right\} .
\end{aligned}
$$

FIG. 1. (a)-(c) Specific heats $C_{\mathrm{v}} / N$ and (d)-(f) ground-state fluctuations as a function of the canonical temperature for systems of $N$ $=100, N=1000$, and $N=10000$ particles. The solid lines represent the results for a spherical trap, the dashed lines for a cube, the circles for a cylinder with a diameter to height ratio of $d / L=1$, the long dashed lines for a box with side lengths $L_{z}=4 L_{y}=4 L_{x}$, and the squares for a cylinder with $d / L=1 / 4$. In all cases the particle density is taken to be $\rho=0.0216 \AA^{-3}$. 
To illustrate the usefulness of our recursion formulas, we consider the ideal gas with parameters of liquid helium in containers of different shapes: (i) a cube with side length $L_{x}, L_{y}, L_{z}$ and energy levels

$$
E_{n_{x}, n_{y}, n_{z}}=\frac{\pi^{2} \hbar^{2}}{2 m_{\mathrm{He}}}\left(\frac{n_{x}^{2}}{L_{x}^{2}}+\frac{n_{y}^{2}}{L_{y}^{2}}+\frac{n_{z}^{2}}{L_{z}^{2}}\right),
$$

(ii) a sphere with radius $a$ and energy levels

$$
E_{n, l}=\frac{\hbar^{2}}{2 m_{\mathrm{He}} a^{2}} u_{n, l}
$$

and degeneracy $\sigma_{n, l}=2 l+1$, and (iii) a cylinder with diameter $d=2 a$, height $L$, and energy levels

$$
E_{n, l, m}=\frac{\hbar^{2}}{2 m_{\mathrm{He}}}\left(\frac{\mathrm{v}_{n, l}^{2}}{a^{2}}+\frac{m^{2} \pi^{2}}{L^{2}}\right)
$$

with $n=1,2, \ldots, l=1,2,3, \ldots$, and $m=\cdots-1,0,-1 \cdots$. We denoted the zeros of the half-integer Bessel functions $J_{n+1 / 2}(r)$ by $u_{n, l}$ and the zeros of the integer Bessel function $J_{n}(r)$ by $\mathrm{v}_{n, l}$.

Figure 1 displays the specific heats and the fluctuations of the ground-state occupation number $\delta \eta_{0} / N$ as a function of the canonical temperature for different trap geometries and $N=100,1000$, and $10000 \mathrm{He}$ atoms. In all cases, the particle density is taken to be $\rho=0.0216 \AA^{-3}$. With growing system size the differences between the specific heats for the different trap geometries almost vanish and approach the typical shape of the curve for the ideal Bose gas. That is, with respect to the specific heat the boundary conditions become more and more unimportant with increasing volume. In contrast, the ground-state fluctuations exhibit a completely different behavior. The cubic box, the compact cylinder with equal diameter and height, and the sphere show almost equal ground-state fluctuations for all system sizes, while the ground-state fluctuations of the stretched box and the stretched cylinder are remarkably larger for temperatures below the critical temperature. This effect is not unexpected because restricting the particle motion in one or two dimensions makes the system act like a lower-dimensional system, which are known to show larger fluctuations. Moreover, the differences between the fluctuations of the stretched traps and the compact traps do not decrease with increasing system size. The reason for this behavior is found in the energy difference between the ground state and the first excited level, which is much larger for the stretched traps than for the compact traps. Since $\delta \eta_{0} / N$ decreases approximately with $N^{-1 / 3}$, the infinite particle number limit is the same for all trap geometries. Under experimental considerations our results imply that the stability of the condensate fraction in anisotropic traps should be considerably smaller than in isotropic traps. In Fig. 1 we plotted $\delta \eta_{0} / N$ to allow good comparison with previous published results $[8,5]$. Since this

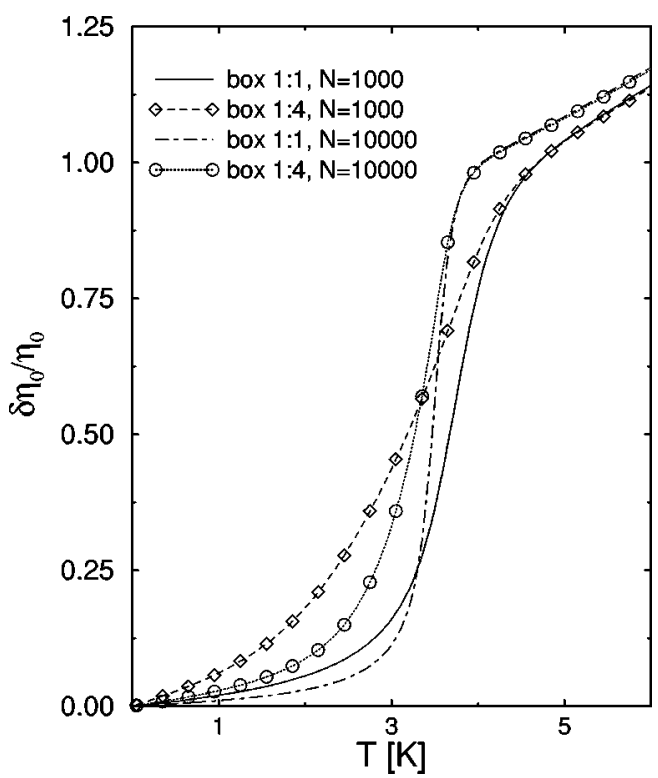

FIG. 2. Relative ground-state fluctuations $\delta \eta_{0} / \eta_{0}$ as a function of temperature for the cubic box and the stretched box and $N$ $=1000$ and 10000 particles.

quantity goes to zero as the system size increases, it is a bad indicator for phase transitions. The relative ground-state fluctuation $\delta \eta_{0} / \eta_{0}$ shown in Fig. 2 is much more conclusive in this respect.

True phase transition only occurs for infinite systems. However, it is well known from other systems, e.g., finite spin lattices and clusters $[11,12]$, that finite systems already display the onset of phase transitions. Instead of having a well defined critical temperature, the transition occurs in a broader crossover region. As can be extracted from Fig. 2, the crossover region, which is indicated by the sharp increase of $\delta \eta_{0} / \eta_{0}$, extends even for the cubic box with 10000 atoms over a temperature range of $0.5 \mathrm{~K}$. For the stretched trap the crossover region is about twice as large.

All calculations have been performed on an IBM-43P $(233 \mathrm{MHz})$ workstation. For 1000 particles a run with 200 temperature points took about six minutes; for 10000 particles it took one hour. A calculation of the same quantities with the recursion formula given in Eq. (1) takes about six hours for 1000 particles (and would take at least 600 hours for 10000 particles). Moreover, due to the numerical instabilities connected with Eq. (1), $N=1000$ was the largest particle number we achieved with this formula, even though we utilized a multiple precision package.

We expect the recursion formulas to be quite useful for calculating the properties of dilute atomic Bose gases in magnetic traps with different geometry. For this purpose we provide an easy to use JAVA program, ${ }^{2}$ which requires as input only the energy level distribution with appropriate degeneracy and calculates the basic properties of finite Bose systems with particle numbers up to 10000 . A slightly faster FORTRAN code is available upon request.

\footnotetext{
${ }^{2}$ The code is available within the World-Wide-Web: http: $\backslash \backslash$ www.physik.uni-oldenburg.de $\backslash \sim$ borrmann $\backslash$ BEC
} 
[1] C. Bradley, C. Sackett, J. Tollet, and R. Hulet, Phys. Rev. Lett. 75, 1687 (1995).

[2] M. Anderson et al., Science 69, 198 (1995).

[3] K. Davies et al., Phys. Rev. Lett. 75, 3969 (1995).

[4] D. Stamer-Kurn et al., Phys. Rev. Lett. 80, 2027 (1998).

[5] S. Grossmann and M. Holthaus, Phys. Rev. Lett. 79, 3557 (1997).
[6] S. Grossmann and M. Holthaus, Opt. Express 1, 262 (1997). [7] P. Navez et al., Phys. Rev. Lett. 79, 1789 (1997).

[8] M. Gajda and K. Rzazewski, Phys. Rev. Lett. 78, 2686 (1997).

[9] P. Borrmann and G. Franke, J. Chem. Phys. 98, 2484 (1993). [10] C. Weiss and M. Wilkens, Opt. Express 1, 272 (1997).

[11] R. E. Kunz and R. S. Berry, Phys. Rev. Lett. 71, 3987 (1993).

[12] D. J. Wales and R. S. Berry, Phys. Rev. Lett. 73, 2875 (1994). 